\title{
The role of venous valves in pressure shielding
}

Constantinos Zervides*, Andrew J Narracott, Patricia V Lawford and

David R Hose

Address: Academic Unit of Medical Physics, School of Medicine and Biomedical Sciences, The University of Sheffield, Sheffield, UK

Email: Constantinos Zervides* - c.zervides@hotmail.co.uk; Andrew J Narracott - a.j.narracott@shef.ac.uk;

Patricia V Lawford - p.lawford@shef.ac.uk; David R Hose - d.r.hose@shef.ac.uk

* Corresponding author

Published: 15 February 2008

BioMedical Engineering OnLine 2008, 7:8 doi:10.1 186//475-925X-7-8

This article is available from: http://www.biomedical-engineering-online.com/content/7/I/8

(c) 2008 Zervides et al; licensee BioMed Central Ltd.

This is an Open Access article distributed under the terms of the Creative Commons Attribution License (http://creativecommons.org/licenses/by/2.0), which permits unrestricted use, distribution, and reproduction in any medium, provided the original work is properly cited.

\begin{abstract}
Background: It is widely accepted that venous valves play an important role in reducing the pressure applied to the veins under dynamic load conditions, such as the act of standing up. This understanding is, however, qualitative and not quantitative. The purpose of this paper is to quantify the pressure shielding effect and its variation with a number of system parameters.
\end{abstract}

Methods: A one-dimensional mathematical model of a collapsible tube, with the facility to introduce valves at any position, was used. The model has been exercised to compute transient pressure and flow distributions along the vein under the action of an imposed gravity field (standing up).

Results: A quantitative evaluation of the effect of a valve, or valves, on the shielding of the vein from peak transient pressure effects was undertaken. The model used reported that a valve decreased the dynamic pressures applied to a vein when gravity is applied by a considerable amount.

Conclusion: The model has the potential to increase understanding of dynamic physical effects in venous physiology, and ultimately might be used as part of an interventional planning tool.

\section{Background}

The motivation behind this study was a desire to understand the physiological effects of compression cuff therapy for prevention of deep vein thrombosis. It is generally accepted [1-4], that deep vein thrombosis is associated with flow stasis, particularly in and around the venous valves and their sinuses. From a survey of the literature, it rapidly became apparent that the role and quantitative performance of venous valves, even in the normal physiological state is poorly understood. Texts on venous physiology always identify the role of the valves as the control of reverse flow [4-7]; most often in the context of muscle pump action to maintain flow in the direction of the heart and sometimes in the context of postural changes and of exercise. The purpose of this paper is to explore the effects of gravity on the pressure and flow distribution in a simple representation of a vein in the leg, and in particular to quantify the role of the valves in pressure shielding under the action of standing. The effects of a range of parameters on the shielding performance of the valves are examined. It is demonstrated that the effects depend not only on the distribution, location and performance of the valves themselves, but also on the geometric and mechanical characteristics of the veins. It is anticipated that this infor- 
mation will be of direct interest to the vascular surgeon because it provides an indication of the likely effect of interventions, including removal or repair of valves as well as insertion of bypass grafts, on peak pressure distributions in the peripheral vasculature.

A person who stands, inactive, for a period of time will be subjected to the full hydrostatic pressure gradient in the venous system and the pressure in the veins in the foot will reach something of the order of $100 \mathrm{mmHg}[8,9,7]$. This is confirmed by Pollack [10] and by Arnoldi [11]. The presence of a valve or valves cannot shield against this static pressure - which will be associated with the physiological phenomenon of blood pooling, and related to oedema through the Starling equation [7], but it can alleviate the transient maximum that will occur as posture is changed. In the absence of valves, a simple analysis would suggest that the transient pressure peak experienced in the vein might be double the final standing pressure. Neglecting inertial and viscoelastic effects in the vessel wall, the stress in the wall of the vein is proportional to the instantaneous applied pressure, and it is postulated that incompetent valves do not provide adequate transient pressure shielding and thus might be strongly implicated in the formation of varicosities $[9,12,13]$. A primary quantitative measure of valve, or rather system, performance is given by its effectiveness in reducing the pressure peaks associated with the transient response. A second measure might be one of the effectiveness of a postural change, and the associated action of gravity on valve opening and closure characteristics and thus on the 'wash out' of the sinuses and displacement of stationary pockets of blood. Although a full three-dimensional analysis is required to address this question in detail, the one-dimensional model presented in this paper is used to examine whether the properties of the system are such that there might be sufficient backflow to close the valve for realistic geometries.

\section{Materials and Methods}

The methodology adopted in this study involves the construction and analysis of a numerical model that is able to capture the spatial and temporal pressure distributions in a collapsible tube that includes valves. The vein is represented as an analogous electrical circuit as illustrated in Figure 1. For simplicity, and because the focus of this study is to capture the characteristic effect of the valves, the vein is represented as a straight collapsible tube. The chosen geometrical configuration is representative of a vein from the lower extremities. The resistive component represents the viscous resistance of the blood, the inductive component the inertia of the blood, and the capacitive component the elasticity (and thus storage capacity) of the vein. A perfect valve is assumed to allow no backflow, and can be represented as a diode (in the analogous

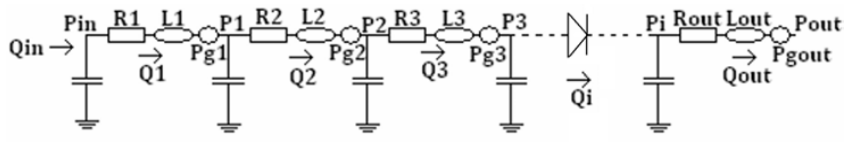

Figure I

Schematic representation of tube model. The resistive component represents the viscous resistance of the blood, the inductive component the inertia of the blood, and the capacitive component the elasticity of the vein. A perfect valve is represented by a diode.

electric circuit a diode allows current to flow in one direction and blocks it from flowing in the opposite direction). A real valve will permit some backflow, associated partly with the swept volume of the valve leaflets during the closure phase, and this is represented by allowing a finite volume of blood to pass through the valve before closure. A 'leaky' valve is assumed to allow a fixed rate of leakage, although this could readily be modified to make the leakage proportional to the pressure drop across the valve segment.

A series of ordinary differential equations are written to represent the electrical system. The nonlinear elastic properties of the vein, including those associated with collapse, are represented by a tube law [14-17]. The main purpose of the tube law is to capture the vein's flexibility at small negative pressures as collapse is initiated, whilst maintaining the properties of a stiffening response for higher negative or positive pressures. A penalty of this formulation is that it does not reduce to the standard linear approximation at small positive pressures, and for the current work, a modification has been implemented to remedy this deficiency. A number of numerical techniques are available for solution of the derived equations [18]. The one adopted for the current study is a Lax Wendroff formulation, which is accurate to second order in time and space. For completeness, the governing equations and the numerical discretisation are listed in Appendix 1. This formulation has been adopted in other studies of the cardiovascular system [19], although Brook [16,20], has identified conditions under which numerical instabilities might be manifest. Numerical testing has indicated that the system is stable under the pertinent conditions for the current study.

One of the important properties of the system that will have significant influence on the results is the boundary conditions applied at the proximal and distal ends of the vein segment. For the purposes of the current study, a constant atmospheric pressure boundary condition has been applied at the proximal end and a constant flow boundary condition at the distal end. It is recognised that the prescribed boundary conditions might represent a gross sim- 
plification of physiological flow in the venous segments of interest. The important feature of the proximal pressure boundary condition is that it allows unimpeded reverse flow into the vein segment as gravity acts. It would be possible to apply a negative pressure representative of that in the thoracic cavity, but as a constant offset this would not significantly affect the results. A transient thoracic pressure representative of respiration could also be applied, but primary focus in this paper is on relatively short term events associated with a near-instantaneous application of gravity. The relatively low frequency respiratory cycle would not significantly modify the results. It has further been assumed that there is a constant flow into the 'bottom' (distal end) of the vein, based on average steady state drainage into the femoral vein. It is unlikely that there will be significant backflow through the distal end during gravity application, due to the higher resistance of the smaller vessels. More sophisticated descriptions of transient flow waveforms measured under a range of conditions can be found in the literature. Of most direct interest is the study reported by Raju $S$ et al [21], who describe flow conditions under ambulatory conditions but not under first application of the gravity field, whilst Neglen and Raju [22] also focus on the measurement of ambulatory pressures in individuals with signs of chronic venous deficiency. Willeput R et al [23] and Abu-Yousef M [24] focus on rest and respiratory conditions. Again, it is suggested that the frequencies associated with these temporal variations are relatively low compared with those associated with the phenomenon addressed in this paper. Furthermore, the starting condition for the analysis is a steady flow through the system (equal to the distal end flow), with no gravity applied.

This paper focuses on the transient pressure and flow distributions in a vein segment, with and without valves, under a near-instantaneous application of gravity. The system is considered passive, and effects of the muscle pump are not included: similarly other relatively low frequency external load factors are neglected. A body force is applied, in the opposite direction to flow, representing the action of gravity under a change of posture from horizontal to vertical: this force is sigmoidal in time, so that there is smooth transition from zero to the full gravity force, which is then held constant.

\section{Results and Discussion}

\section{Baseline condition, no valve}

A series of numerical tests were performed, to ensure that the model performed properly and returned accurate results for simple conditions, including for example using a linear tube law, for which analytical comparisons were available, and for other conditions for which numerical results have been published $[17,19]$. Once these tests were passed, a first baseline analysis was performed using the following parameters: vein diameter $1.19 \mathrm{~cm} \mathrm{[25],} \mathrm{vein}$ thickness-to-diameter ratio 0.2 [26], vein length $1 \mathrm{~m}$, wall stiffness $1 \mathrm{MPa}$ [27], blood viscosity 0.004 Pa.s, blood

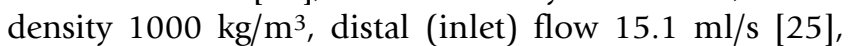
proximal (outlet) pressure $0 \mathrm{mmHg}(0 \mathrm{~Pa})$, near instantaneous body-force application (gravity increased from zero to $9.8 \mathrm{~m} / \mathrm{s}^{2}$ over 0.01 milliseconds). These values are given in convenient units: all analyses were performed in SI units. The initial condition, prior to the application of gravity, was a steady flow in the opposite direction to that in which gravity would be applied (i.e from distal to proximal end of the tube).

Discretisation-independence tests were performed to ensure that the results did not depend either on the number of elements used to represent the geometry of the vessel or on the simulation time-step. Results for the baseline condition and for several parameter variations are reported in Table 1. One of the most important results is the 'dynamic pressure ratio'. This is defined as the ratio of the peak dynamic pressure to the unavoidable static pressure that will be reached when the system has stabilised. Also reported in Table 1 are the magnitudes of the first and second pressure peaks recorded as the system oscillates (to give an indication of how quickly the overall peak is reached), the time for which the valve remains closed during the first oscillatory phase, and measures of the peak positive and negative area changes as the vein expands and collapses.

Figure 2 presents the computed pressure against time at the distal end of the vessel. The system is oscillatory (the only damping in this system is that due to the viscosity of the blood - it is recognised that the real system will have additional damping due to the viscoelastic properties of the vessel wall, and probably more importantly of the surrounding tissues), but after a period of approximately 12 $\mathrm{s}$ the pressure at the distal end remains within $2 \%$ of the steady state value at $74.5 \mathrm{mmHg}(9936.3 \mathrm{~Pa})$, consistent with the hydrostatic force applied plus the (small) pressure drop associated with the superimposed steady flow. The overshoot associated with the dynamic system produces a peak pressure of $136.5 \mathrm{mmHg}(18151 \mathrm{~Pa})$, representing a dynamic pressure ratio of 1.83 (a simple first principles analysis without damping would suggest a ratio of 2.00 [28], so this result is plausible).

Figure 3 illustrates the pressure and flow against time at a point halfway along the vein. The pressure exhibits similar characteristics to that at the distal end, oscillating about its hydrostatic condition of one-half of the distal end value. The flow starts from the initial condition, oscillates in response to the sudden application of gravity, and returns to the steady condition after approximately $12 \mathrm{~s}$. It is noted that there is very significant reverse flow $(\approx 30 \mathrm{ml} / \mathrm{s})$ 
Table I: Baseline condition, valve performance and gravity application time results

\begin{tabular}{|c|c|c|c|c|c|}
\hline Parameters & No valve & Perfect valve & "Real" valve & Leaky valve & Gravity test \\
\hline Diameter $(\mathrm{cm})$ & 1.2 & 1.2 & 1.2 & 1.2 & 1.2 \\
\hline Length $(\mathrm{m})$ & I & I & 1 & 1 & 1 \\
\hline Young's modulus (kPa) & 1000 & 1000 & 1000 & 1000 & 1000 \\
\hline Poisson's ratio & 0.5 & 0.5 & 0.5 & 0.5 & 0.5 \\
\hline Blood viscosity (mPas) & 4 & 4 & 4 & 4 & 4 \\
\hline Blood density $\left(\mathrm{Kgm}^{-3}\right)$ & 1000 & 1000 & 1000 & 1000 & 1000 \\
\hline Gravity application time (s) & Near instantaneous & Near instantaneous & Near instantaneous & Near instantaneous & 0.1 \\
\hline Valve distribution & No valve & One & One & One & One \\
\hline Valve location from inlet $(\mathrm{m})$ & No valve & 0.5 & 0.5 & 0.5 & 0.5 \\
\hline Valve performance & No valve & Perfect & "Real" & Leaky & Perfect \\
\hline First peak (mmHg) & I36.9 (I8.2 kPa) & $60.2(8.01 \mathrm{kPa})$ & $60.3(8.02 \mathrm{kPa})$ & $65.4(8.69 \mathrm{kPa})$ & $50.3(6.69 \mathrm{kPa})$ \\
\hline Second peak $(\mathrm{mmHg})$ & $127.1(16.9 \mathrm{kPa})$ & $81.9(10.9 \mathrm{kPa})$ & $81.9(10.9 \mathrm{kPa})$ & II $5.8(15.4 \mathrm{kPa})$ & $72.6(9.66 \mathrm{kPa})$ \\
\hline Maximum pressure $(\mathrm{mmHg})$ & $136.9(\mid 8.2 \mathrm{kPa})$ & $93.3(\mathrm{I} 2.4 \mathrm{kPa})$ & $93.9(12.4 \mathrm{kPa})$ & II $5.8(I 5.4 \mathrm{kPa})$ & $99.3(13.2 \mathrm{kPa})$ \\
\hline Dynamic pressure ratio & 1.83 & 1.25 & 1.25 & 1.51 & $\mathrm{I} .33$ \\
\hline Valve closed time (s) & No valve & 0.17 & 0.17 & 0.11 & 0.16 \\
\hline Maximum collapse (\%) & 0.02 & 20.2 & 20.4 & 3.57 & 8.17 \\
\hline Maximum expansion (\%) & 15.1 & 9.89 & 9.89 & 12.5 & 10.6 \\
\hline
\end{tabular}

occurring approximately $80 \mathrm{~ms}$ after gravity is applied. The reverse flow acts to fill the more distal sections of the vein as it distends under the increased (gravitational) pressure. As demonstrated later, this reverse flow would be enough to close a competent valve and consequently to shield the lower parts of the vein and reduce peak dynamic pressures. If there were sufficient inflow from below (unlikely in the human system unless the process of standing is done very slowly) then the vein could be

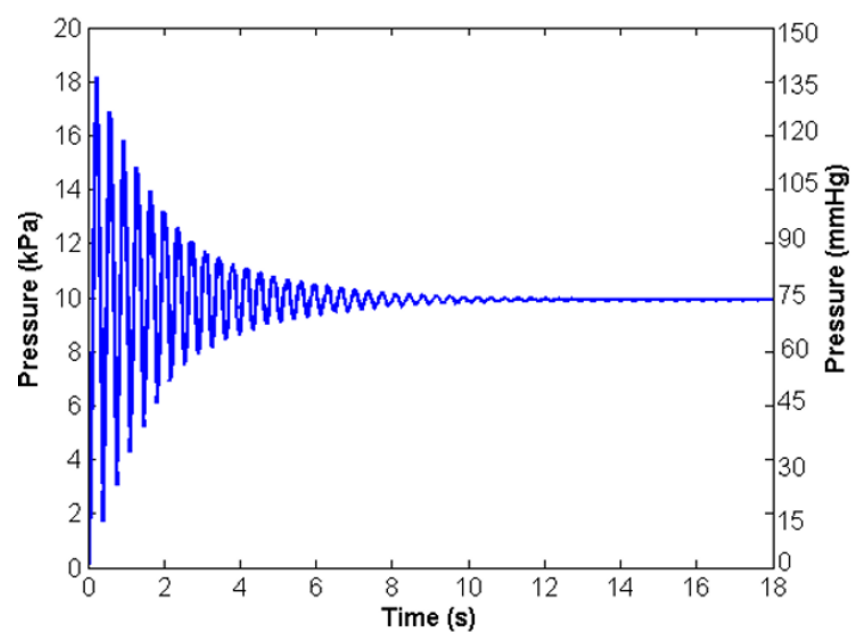

Figure 2

Baseline condition pressure against time at the distal end of the vessel. The system is oscillatory but after approximately $12 \mathrm{~s}$ the pressure at the distal end remains within $2 \%$ of the steady state value at $74.5 \mathrm{mmHg}(9936.3$ $\mathrm{Pa}$ ), consistent with the hydrostatic force applied plus the (small) pressure drop associated with the superimposed steady flow. filled (distended) entirely by the inflow, and reverse flow might not occur.

The analysis of the baseline condition gives some confidence in the operation of the model, and also provides quantitative information on the peak pressure that can be expected at the distal end of the vein in the absence of protection from venous valves. Further confidence has been developed by comparison of the results with those from three dimensional models using a commercial finite ele-
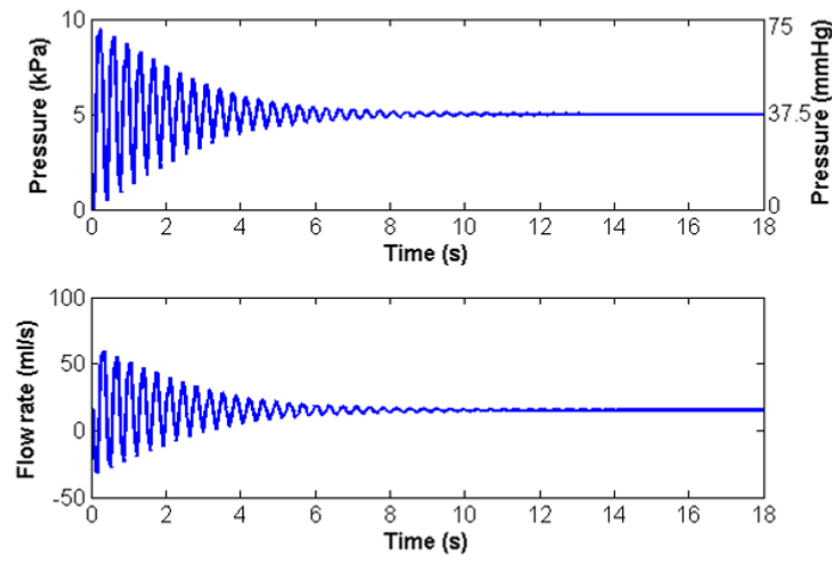

Figure 3

Baseline condition pressure against time and flow rate against time at the midpoint of the vessel. The pressure exhibits similar characteristics to that at the distal end, oscillating about its hydrostatic condition of one-half of the distal end value. The flow starts from the initial condition, oscillates in response to the sudden application of gravity, and returns to the steady condition after approximately $12 \mathrm{~s}$. 
ment code, but the reporting of these results is beyond the scope of this paper.

\section{Effect of perfect, real and incompetent valves}

The model was next used to evaluate and to provide quantitative information about a hypothesis often expressed in text book descriptions of venous physiology, e.g. Browse [13]:

'The venous valves normally protect the wall of the vein below each valve from the pressure in the vein above it.'

A perfect (no reverse flow) valve was introduced into the vein at a point halfway along its length, and a simulation performed to illustrate the effect of the valve on the peak pressures and flows in the system. The oscillatory nature of the system is such that the valve will open and close several times before finally settling in the open state in the hydrostatic condition. Attention is focused on the early phase, from the time of gravity application through to the time of peak pressure in the system. Figure 4 illustrates the pressure at the distal end against time during the first second after application of gravity, together with the results for the case with no valve. The distinctive saw-tooth appearance of the oscillations is due to the summation of pressure waves as they reflect from the domain boundaries. The presence of the valve significantly changes the form of the dynamic response. Without the valve, the system undergoes relatively high-amplitude oscillations about the hydrostatic pressure value, gradually damping towards the steady state. With the valve, the approach

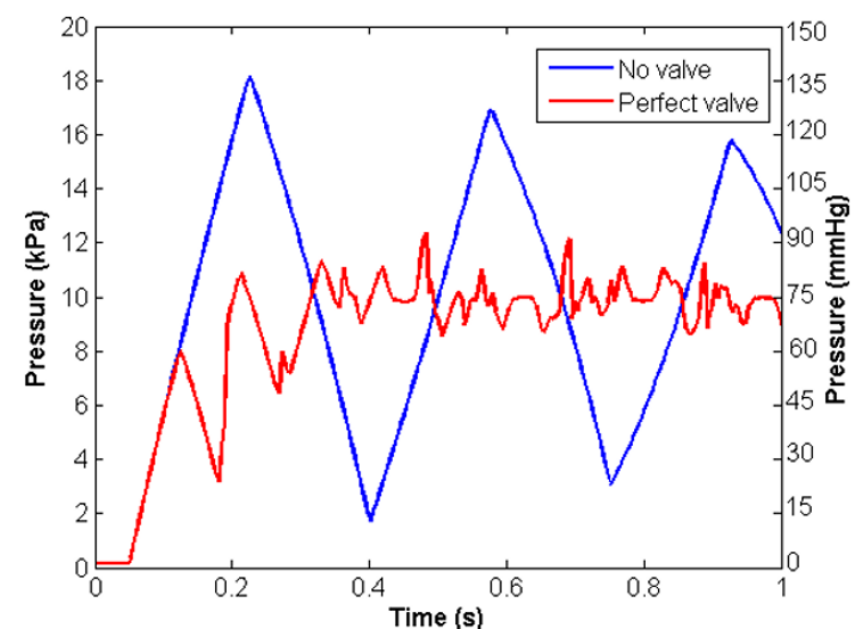

Figure 4

Pressure against time at the distal end of the vessel with and without perfect valve. The presence of the valve significantly changes the form of the dynamic response and the approach towards the steady state is reasonably asymptotic, with relatively lower pressure oscillations superimposed on the asymptote. towards the steady state is reasonably asymptotic, with relatively lower pressure oscillations superimposed on the asymptote. The peak pressure for the system with a valve is $93.0 \mathrm{mmHg}(12370 \mathrm{~Pa})$, representing a dynamic pressure ratio of 1.25. Perhaps, therefore, the most important observation is that, consistent with the hypothesis, the valve has provided a very significant shielding effect (over $45 \mathrm{mmHg}$ (5985 Pa) reduction in peak pressure).

Figure 5 illustrates pressure and flow at the section immediately distal to the valve, together with the no-valve system. Here the effect of the valve is very clearly indicated. The analyses are identical up to the point at which flow reversal occurs. At this point, the valve closes and the flow is set to zero until forward flow is re-established, partly by the constant influx from the distal boundary and partly by wave reflection. The segment of vein distal to the valve now acts as a closed cylinder (at least in the portion immediately distal to the valve which takes some time to be affected by the constant influx from the (relatively distant) distal boundary). Blood continues to fall towards the distal end but at a significantly reduced rate, and the segment immediately distal to the valve reduces in diameter and starts to collapse. The degree of collapse is determined by the rate of application of gravity and the physical characteristics of the system. In the model reported here, the area reduction immediately distal to the valve is approximately $20 \%$. Figure 6 illustrates the ratio of cross-sectional area to undeformed cross-sectional area along the length of the vein at different points in time and Figure 7 illustrates the pressure variation along the length of the vein at different points in time, showing clearly the pressure discontinuity at the valve. The presence of waves reflecting from proximal and distal boundaries is also apparent. After approximately $6.3 \mathrm{~s}$, the system settles to within $2 \%$ of the hydrostatic state.

The above results illustrate the effect of a perfect valve on pressure shielding. A real valve must permit some reverse flow as it is swept to the closed position. A first estimate of the volume of flow reversal (neglecting the reverse flow as the valve is first entrained) can be made by measuring the swept volume of the valve during closure. An estimate of this volume, based on conic sections is $0.12 \mathrm{ml}$. Results based on this approximation are illustrated in Figure 8, together with those for the perfect valve. Although the 'real' valve allows some regurgitation as the leaflets are swept to closure, the volume associated with this event is small and the effect is negligible; this might not be true if a larger regurgitant volume were to be admitted, reflecting the entrainment of the leaflets in the reversing flow. An incompetent valve might be expected to lie further towards the no-valve condition. To test this hypothesis, an analysis has been performed in which the negative flow rate through the valve has been limited to $20 \mathrm{ml} / \mathrm{s}$. In this 

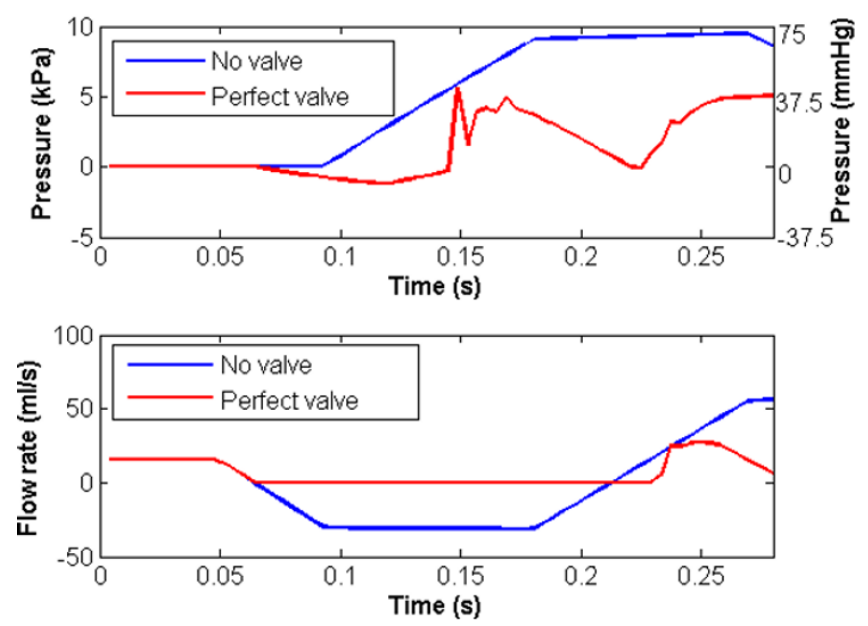

Figure 5

Pressure against time and flow rate against time at the midpoint of the vessel with and without a perfect valve. Here the effect of the valve is very clearly indicated since the analyses are identical up to the point at which flow reversal occurs.

condition, the pressure shielding effect is substantially reduced, and the dynamic pressure ratio is increased (from 1.25 for the perfect valve) to 1.51 .

In a final test on the reference configuration with a perfect valve, the time over which gravity was applied was increased from near instantaneous to $100 \mathrm{~ms}$ (more consistent with the likely time taken to stand up). As

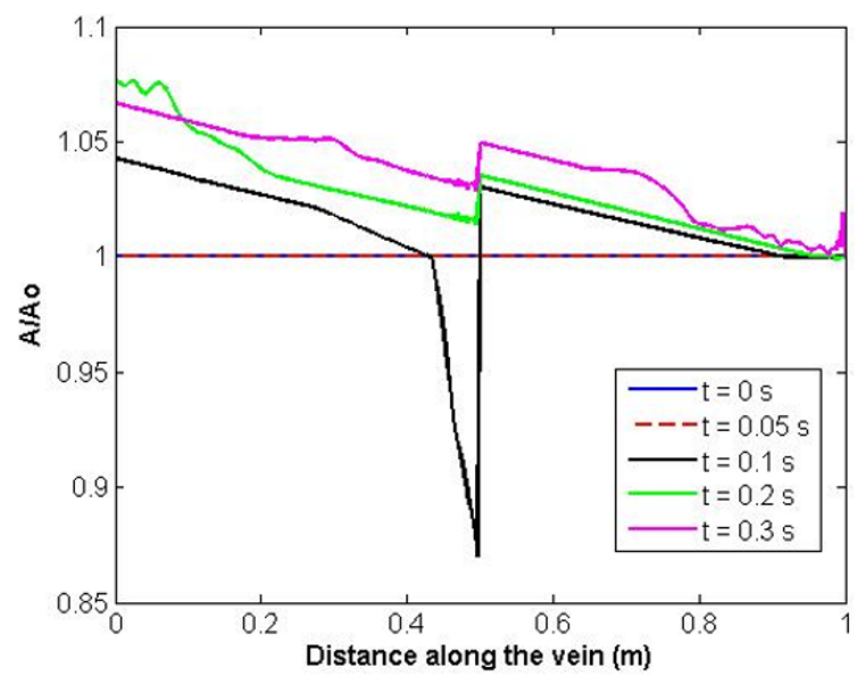

Figure 6

Ratio of cross-sectional area over undeformed cross sectional area along the vein length for increasing time.

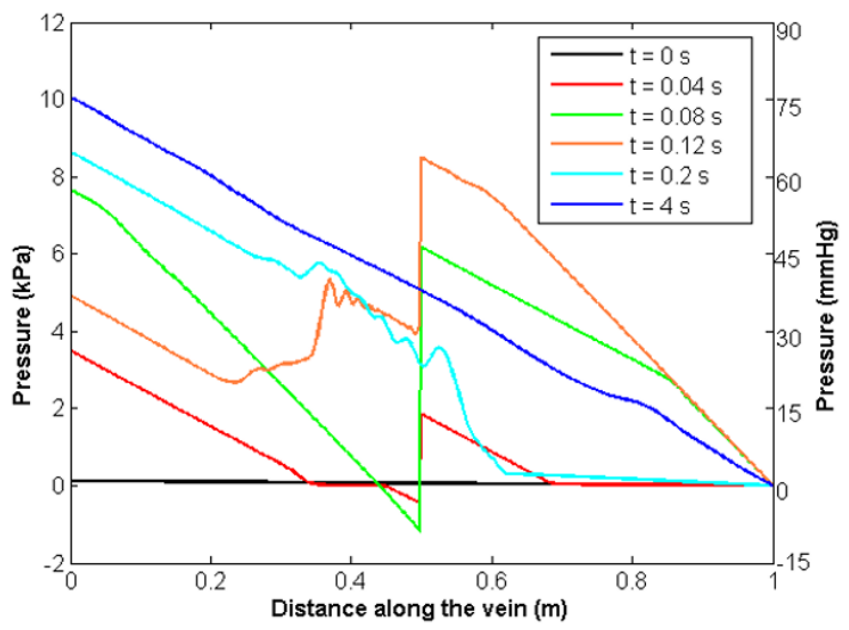

Figure 7

Pressure along the vein length for increasing time showing a pressure discontinuity at the valve location.

expected, the first and second pressure peaks were lower (by the order of 10\%) but perhaps surprisingly, the absolute peak was a little higher. This was due to different interactions of the wave reflections in the system, but it does not affect the overall shape of the response, nor indeed the conclusions.

\section{Parameter studies}

The model permits the examination of the effect of change of the geometrical and mechanical characteristics of the system on the pressure-shielding phenomenon. Results for variations of a number of parameters are presented in

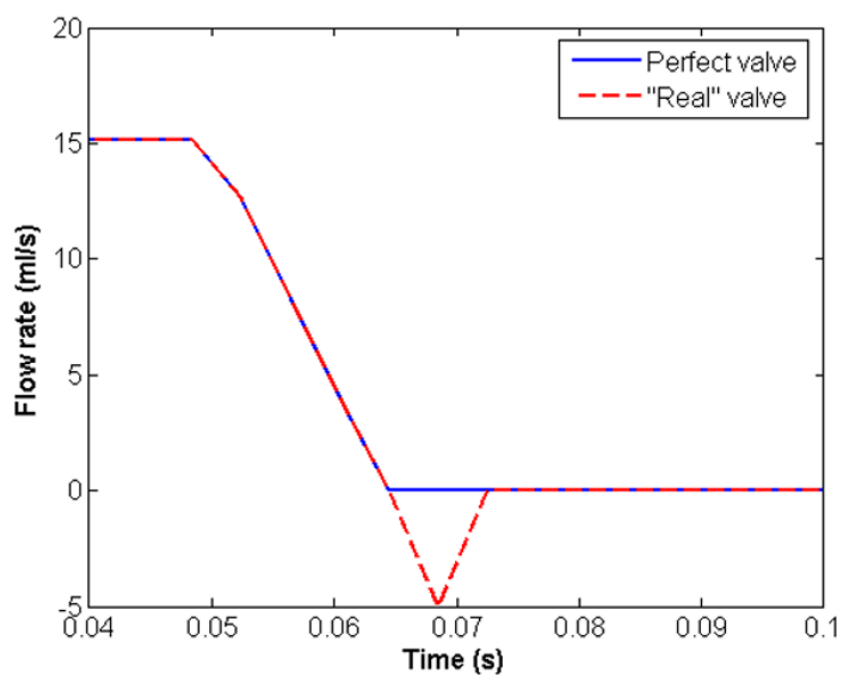

Figure 8

Flow rate vs time through a perfect and "real" valve. 
Table 2: Parameter variation test results

\begin{tabular}{|c|c|c|c|c|c|c|c|c|c|c|}
\hline Parameters & Perfect valve & \multicolumn{2}{|c|}{ Diameter test } & \multicolumn{2}{|c|}{ Stiffness test } & Distribution test & \multicolumn{2}{|c|}{ Length test } & \multicolumn{2}{|c|}{ Location test } \\
\hline Diameter $(\mathrm{cm})$ & 1.2 & 0.8 & 1.6 & & & 1.2 & & .2 & I & \\
\hline Length $(m)$ & I & \multicolumn{2}{|c|}{1} & \multicolumn{2}{|c|}{1} & 1 & 0.50 & 0.75 & \multicolumn{2}{|c|}{1} \\
\hline Young's modulus (kPa) & 1000 & \multicolumn{2}{|c|}{1000} & \multicolumn{2}{|r|}{2000} & 1000 & \multicolumn{2}{|c|}{1000} & \multicolumn{2}{|c|}{1000} \\
\hline Poisson's ratio & 0.5 & \multicolumn{2}{|c|}{0.5} & \multicolumn{2}{|c|}{0.5} & 0.5 & \multicolumn{2}{|c|}{0.5} & \multicolumn{2}{|c|}{0.5} \\
\hline Blood viscosity (mPas) & 4 & \multicolumn{2}{|c|}{4} & \multicolumn{2}{|c|}{4} & 4 & \multicolumn{2}{|c|}{4} & \multicolumn{2}{|c|}{4} \\
\hline Blood density $\left(\mathrm{Kgm}^{-3}\right)$ & 1000 & \multicolumn{2}{|c|}{1000} & \multicolumn{2}{|c|}{1000} & 1000 & \multicolumn{2}{|c|}{1000} & \multicolumn{2}{|c|}{1000} \\
\hline Gravity application time (s) & Near instantaneous & \multicolumn{2}{|c|}{ Near instantaneous } & \multicolumn{2}{|c|}{ Near instantaneous } & Near instantaneous & \multicolumn{2}{|c|}{ Near instantaneous } & \multicolumn{2}{|c|}{ Near instantaneous } \\
\hline Valve distribution & One & \multicolumn{2}{|c|}{ One } & \multicolumn{2}{|c|}{ One } & Two & \multicolumn{2}{|c|}{ One } & \multicolumn{2}{|c|}{ One } \\
\hline Valve location from inlet $(m)$ & 0.5 & \multicolumn{2}{|c|}{0.5} & \multicolumn{2}{|c|}{0.5} & 0.25 and 0.75 & \multicolumn{2}{|c|}{0.5} & 0.25 & 0.75 \\
\hline Valve performance & Perfect & \multicolumn{2}{|c|}{ Perfect } & & & Perfect & & fect & Per & \\
\hline First peak $(\mathrm{mmHg})$ & $60.2(8.01 \mathrm{kPa})$ & $66.3(8.81 \mathrm{kPa})$ & $56.8(7.55 \mathrm{kPa})$ & $56.3(7.48 \mathrm{kPa})$ & $63.2(8.4 \mathrm{kPa})$ & $32.6(4.34 \mathrm{kPa})$ & $33.2(4.4 \mathrm{I} \mathrm{kPa})$ & $46.8(6.23 \mathrm{kPa})$ & $32.6(4.33 \mathrm{kPa})$ & $87.2(11.6 \mathrm{kPa})$ \\
\hline Second peak $(\mathrm{mmHg})$ & $81.9(10.9 \mathrm{kPa})$ & II $4.3(15.2 \mathrm{kPa})$ & $74.9(9.97 \mathrm{kPa})$ & $74.6(9.92 \mathrm{kPa})$ & $95.5(12.7 \mathrm{kPa})$ & $55.6(7.39 \mathrm{kPa})$ & $55.9(7.44 \mathrm{kPa})$ & $57(7.58 \mathrm{kPa})$ & $99.3(13.2 \mathrm{kPa})$ & $90.2(12 \mathrm{kPa})$ \\
\hline Maximum pressure $(\mathrm{mmHg})$ & $93.3(12.4 \mathrm{kPa})$ & $115.8(15.4 \mathrm{kPa})$ & $89.5(11.9 \mathrm{kPa})$ & $90.9(12.1 \mathrm{kPa})$ & $104.5(13.9 \mathrm{kPa})$ & $92.9(12.36 \mathrm{kPa})$ & $57.2(7.61 \mathrm{kPa})$ & $77.5(10.31 \mathrm{kPa})$ & II0.5 (14.7 kPa) & $105.3(14 \mathrm{kPa})$ \\
\hline Dynamic pressure ratio & 1.25 & 1.47 & 1.22 & 1.21 & 1.41 & 1.25 & 1.53 & 1.39 & 1.49 & 1.41 \\
\hline Valve closed time (s) & 0.17 & 0.11 & 0.19 & 0.26 & 0.11 & 0.09 and 0.17 & 0.06 & 0.11 & 0.16 & 0.15 \\
\hline Maximum collapse (\%) & 20.2 & 3.64 & 24.5 & 48.2 & 6.06 & 35.6 & 3.58 & 10.6 & 3.25 & 42.3 \\
\hline Maximum expansion (\%) & 9.89 & 12.1 & 9.87 & 21.2 & 5.41 & 9.89 & 5.92 & 7.19 & 11.9 & 11.5 \\
\hline
\end{tabular}


Table 2. Reducing the undeformed diameter of the vein by one-third increases the dynamic pressure ratio to 1.47 in the presence of the valve, and increasing the diameter of the vein by one third reduces the peak pressure ratio to 1.22. Similarly, doubling the vein stiffness causes an increase to 1.41, and halving it causes a decrease to 1.21. Each of these results is qualitatively consistent with expectations based on an understanding of the physics phenomena: the model provides quantification of the effect.

Changing the overall length of the system (whilst maintaining the position of the valve at $0.5 \mathrm{~m}$ from the inlet), or changing the position of the valve along the length of the $1 \mathrm{~m}$ vein, increased the dynamic pressure ratio, suggesting that the optimal position for a valve in a vein with the imposed boundary conditions is near to the midpoint. Finally, a test with two valves, one at one-quarter length and one at three-quarters length produced a pressure shielding of the same magnitude as that obtained with a single valve halfway along the vein.

\section{Conclusion}

The one-dimensional model reported in this paper permits the quantitative evaluation of the effects of venous valves on the loads and geometrical changes induced by the action of gravity. It is an important first step in a longer-term study of venous valves, venous diseases and their prevention. With refinements to the venous valve description, applied tube law and boundary conditions, a more physiologically realistic model can be created in an equivalent form to the Westerhof arterial model [29]. This will enable the model to be validated against physiological data. For the purposes of this paper though the greatest interest is in the dynamic pressure ratio, which provides a measure of the increase of the peak local pressure in the system (due to dynamic effects) over the corresponding hydrostatic pressure. It is demonstrated that, for a configuration typical of the femoral vein, the dynamic pressure ratio without a valve is 1.83 , and that with a perfect valve located halfway along the vein is 1.25 . The absolute pressure reduction is over $40 \mathrm{mmHg}(5320 \mathrm{~Pa})$. The model has been used to investigate the quantitative influence of variation of a number of parameters. Following extensive in vitro and in vivo validation, this model might be used to evaluate the effects of valve incompetence on venous pressure distributions and could have implications for the understanding of the progression of disease in the context of varicose veins. It might also be used as part of an interventional planning tool.

The reported study is entirely theoretical. Validation against other reported numerical studies has been performed to give confidence in the numerical implementation, but validation against experimental data is an important next step. Some experimental data does exist for collapsible tubes with gravity effects, for example of the filling under gravity of an initially collapsed tube [30], but none of direct relevance to the current study. A preliminary experimental model that can be used directly to validate the current model has been reported by Potter [31] and Burnett [32] but this is not yet sufficiently mature for detailed comparative evaluation. Validation against physiological data, such as that presented in the works referenced in the section on boundary conditions, will require first the construction of an improved numerical model with a more complex network representation of the venous circulation in the lower limb. In the longer term a detailed three dimensional (3D) model is required to compute the haemodynamic characteristics in the region of the valve, and to evaluate the effects of local geometric and material variations. The $1 \mathrm{D}$ model described in this paper provides important mutual validation data for such a 3D model, as well as the potential to provide local boundary conditions for it in the region of the valve.

\section{Competing interests}

The author(s) declare that this work was funded by the British Heart Foundation which also financed this manuscript.

\section{Authors' contributions}

CZ carried out all of the computational work reported in this manuscript. DRH drafted the framework of the manuscript, integrated the computational results provided by $\mathrm{CZ}$ and drafted the conclusions. DRH, PVL and AJN conceived the program of work, supervised its progression and provided distinct and separate intellectual input. All authors participated in the critical review and revision of this manuscript prior to submission.

\section{Appendix: Equations and discretisation Al: Governing equations}

The continuity equation used was:

$$
C^{\prime} \frac{\partial P}{\partial t}+\frac{\partial Q}{\partial z}=0
$$

where

$$
C_{i}^{\prime n}=\frac{d A^{n}}{d P_{i}} l=\frac{1}{\left(10 K p\left(\frac{\left(A_{i}^{n}\right)^{9}}{A_{o}^{10}}\right)+1.5 K p\left(\frac{\left(A_{o}^{1.5}\right)}{\left.\sqrt{\left(A_{i}^{n}\right)}\right)}\right)\right.}
$$

The momentum equation used was: 


$$
L^{\prime} \frac{\partial Q}{\partial t}+\frac{\partial P}{\partial z}=-R^{\prime} Q
$$

where

$$
\begin{aligned}
R_{i}^{\prime n} & =\frac{8 \pi \mu}{\left(A_{i}^{n}\right)^{2}} \\
L_{i}^{m} & =\frac{\rho}{\left(A_{i}^{n}\right)}
\end{aligned}
$$

The tube law used was:

$$
P_{i}^{n}-P_{e}=K p\left(\left(\frac{A_{i}^{n}}{A_{o}}\right)^{z}-\left(\frac{A_{i}^{n}}{A_{o}}\right)^{-1.5}\right)
$$

where

$$
K p=\frac{E}{12\left(1-\sigma^{2}\right)}\left(\frac{h}{r}\right)^{3}
$$

The variables used in equations 1-7 were: $A_{0}$ - undeformed vessel area-, $\mathrm{A}_{\mathrm{i}}$ - vessel area at point $\mathrm{i}$ along the vessel length at time $n-, \mu$ - fluid viscosity-, $\rho$ - fluid density-, E - vessel wall Young's modulus, $\sigma$-vessel Poisson's ratio, $\mathrm{h}$ - vessel wall thickness and finally $\mathrm{r}$ - vessel radius. All variables used were described in SI units.

\section{A2: Lax-Wendroff discretisation}

To find the two equations of interest for pressure and flow using the Lax-Wendroff technique the following two equations have to be used.

$$
\begin{gathered}
P_{i}^{t+\Delta t}=P_{i}^{t}+\Delta t\left(\frac{\partial P}{\partial t}\right)_{i}+\frac{\Delta t^{2}}{2}\left(\frac{\partial^{2} P}{\partial t^{2}}\right)_{i} \\
Q_{i}^{t+\Delta t}=Q_{i}^{t}+\Delta t\left(\frac{\partial Q}{\partial t}\right)_{i}+\frac{\Delta t^{2}}{2}\left(\frac{\partial^{2} Q}{\partial t^{2}}\right)_{i}
\end{gathered}
$$

The above two equations are the Taylor series expansion of second order accuracy. Based on the above two equations and the simplified version of the mass and momentum conservation equations above, the equations needed for pressure and flow using the Lax-Wendroff technique can be found. Firstly for pressure to be found the first and second time derivatives of pressure have to be found. The first one is easily found from the continuity equation and is:

$$
C^{\prime} \frac{\partial P}{\partial t}+\frac{\partial Q}{\partial x}=0 \Rightarrow \frac{\partial P}{\partial t}=-\frac{1}{C^{\prime}} \frac{\partial Q}{\partial x}
$$

In order to find the second time derivative of pressure the first time derivative of pressure must be differentiated in time. This gives:

$$
\frac{\partial P}{\partial t}=-\frac{1}{C^{\prime}} \frac{\partial Q}{\partial x} \Rightarrow \frac{\partial}{\partial t}\left(\frac{\partial P}{\partial t}\right)=-\frac{1}{C^{\prime}} \frac{\partial}{\partial t}\left(\frac{\partial Q}{\partial x}\right) \Rightarrow \frac{\partial^{2} P}{\partial t^{2}}=-\frac{1}{C^{\prime}} \frac{\partial^{2} Q}{\partial t \partial x}
$$

Thus to find the second time derivative of pressure $\frac{\partial^{2} Q}{\partial t \partial x}$ must be found. This is done by differentiating the momentum equations in space. This gives:

$$
\begin{aligned}
& L^{\prime} \frac{\partial Q}{\partial t}+\frac{\partial P}{\partial x}=-R^{\prime} Q \Rightarrow \frac{\partial Q}{\partial t}=-\frac{1}{L^{\prime}} \frac{\partial P}{\partial x}-\frac{R^{\prime}}{L^{\prime}} Q \Rightarrow \frac{\partial}{\partial x}\left(\frac{\partial Q}{\partial t}\right)=-\frac{1}{L^{\prime}} \frac{\partial}{\partial x}\left(\frac{\partial P}{\partial x}\right)-\frac{R^{\prime}}{L^{\prime}} \frac{\partial}{\partial x}(Q) \\
& \Rightarrow \frac{\partial^{2} Q}{\partial t \partial x}=-\frac{1}{L^{\prime}} \frac{\partial^{2} P}{\partial x^{2}}-\frac{R^{\prime}}{L^{\prime}} \frac{\partial Q}{\partial x}
\end{aligned}
$$

Now by substituting 12 in 11 the second time derivative of pressure is found to be:

$$
\frac{\partial^{2} P}{\partial t^{2}}=-\frac{1}{C^{\prime}} \frac{\partial^{2} Q}{\partial t \partial x} \Rightarrow \frac{\partial^{2} P}{\partial t^{2}}=-\frac{1}{C^{\prime}}\left[-\frac{1}{L^{\prime}} \frac{\partial^{2} P}{\partial x^{2}}-\frac{R^{\prime}}{L^{\prime}} \frac{\partial Q}{\partial x}\right]
$$

Since both the first and second time derivative of pressure are found, equation 8 can be solved. This gives:

$$
\begin{aligned}
& P_{i}^{t+\Delta t}=P_{i}^{t}+\Delta t\left(\frac{\partial P}{\partial t}\right)_{i}+\frac{\Delta t^{2}}{2}\left(\frac{\partial^{2} P}{\partial t^{2}}\right)_{i} \Rightarrow \\
& P_{i}^{t+\Delta t}=P_{i}^{t}+\Delta t\left[-\left.\frac{1}{C^{\prime}} \frac{\partial Q}{\partial x}\right|_{i}\right]+\frac{\Delta t^{2}}{2}\left[-\frac{1}{C^{\prime}}\left[-\left.\frac{1}{L^{\prime}} \frac{\partial^{2} P}{\partial x^{2}}\right|_{i}-\left.\frac{R^{\prime}}{L^{\prime}} \frac{\partial Q}{\partial x}\right|_{i}\right]\right] \Rightarrow \\
& P_{i}^{t+\Delta t}=P_{i}^{t}-\left.\frac{\Delta t}{C^{\prime}} \frac{\partial Q}{\partial x}\right|_{i}+\frac{\Delta t^{2}}{2 C^{\prime} L^{\prime}}\left[\left.\frac{\partial^{2} P}{\partial x^{2}}\right|_{i}\right]+\left.\frac{\Delta t^{2} R^{\prime}}{2 C^{\prime} L^{\prime}} \frac{\partial Q}{\partial x}\right|_{i} \Rightarrow \\
& P_{i}^{t+\Delta t}=P_{i}^{t}+\frac{\Delta t^{2}}{2 C L}\left[P_{i+1}^{t}-2 P_{i}^{t}+P_{i-1}^{t}\right]-\frac{\Delta t}{2 C}\left[Q_{i+1}^{t}-Q_{i-1}^{t}\right]+\frac{\Delta t^{2} R^{\prime}}{2 C L^{\prime}}\left[Q_{i}^{t}-Q_{i-1}^{t}\right]
\end{aligned}
$$

For flow to be found the same methodology as used to find the pressure equation is used and gives:

$Q_{i}^{t+\Delta t}=Q_{i}^{t}\left(1-\frac{\Delta t R^{\prime}}{L^{\prime}}\right)-\frac{\Delta t}{2 L}\left(P_{i+1}^{t}-P_{i-1}^{t}\right)+\frac{\Delta t^{2}}{2 L C}\left[Q_{i+1}^{t}-2 Q_{i}^{t}+Q_{i-1}^{t}\right]+\frac{\Delta t^{2} R^{\prime}}{2 L L^{\prime}}\left(P_{i+1}^{t}-P_{i}^{t}\right)+Q_{i}^{t} \frac{\Delta t^{2} R^{\prime 2}}{2 L^{\prime 2}}$ 


\section{Acknowledgements}

This study was funded by the British Heart Foundation under BHF PhD studentship FS/05/086/19462. Many useful synergies arose between this and a parallel study on deep vein thrombosis, EPSRC GR/S86464/0I, funded by the Engineering and Physical Sciences Research Council.

\section{References}

I. Gibbs NM: Venous thrombosis of the lower limbs with particular reference to bed-rest. Br J Surg 1957, 45:209-236.

2. McLachlin AD, McLachlin JA, Jory TA, Rawling EG: Venous stasis in the lower extremities. Ann Surg 1960, 152:678-685.

3. Sevitt $\mathrm{S}$ : The structure and growth of valve-pocket thrombi in femoral veins. J Clin Pathol 1974, 27:5 17-528.

4. Gottlob R, May R, Geleff S: Venous valves: morphology, function, radiology, surgery. New York: Springer-Verlag; 1986.

5. Tooke JE, Lowe GO: A textbook of vascular medicine. London , Arnold; 1996

6. Guyton AC, Hall JE: Textbook of medical physiology. Philadelphia, Saunders; 2000

7. Klabunde RE: Cardiovascular physiology concepts. Philadelphia , Lippincott Williams and Wilkins; 2005.

8. Stick C, Jaeger H, Witzleb E: Measurements of volume changes and venous pressure in the human lower leg during walking and running. I Appl Physiol 1992, 72:2063-2068.

9. Tibbs DJ: Varicose veins and related disorders. Boston, Butterworth-Heinemann; 1992.

10. Pollack AA, Wood EH: Venous pressure in the saphenous vein at the ankle in man during exercise and changes in posture. Journal of Applied Physiology 1949, 1:649-662.

II. Arnoldi CC: Venous pressure in the leg of healthy human subjects at rest and during muscular exercise in the nearly erect position. Acta Chir Scand 1965, 130:570-583.

12. Tibbs DJ: Varicose veins, venous disorders, and lymphatic problems in the lower limbs. New York, Oxford University Press; 1997.

13. Browse NL: Diseases of the veins. New York, Arnold ; 1999.

14. Shapiro AH: Steady Flow in Collapsible Tubes. J Biomech Eng 1977, 99:126-147.

15. Elad D, Kamm RD, Shapiro $A H$ : Choking phenomena in a lunglike model. J Biomech Eng 1987, 109: I-9.

16. Brook BS: The effect of gravity on the haemodynamics of the giraffe jugular vein. University of Leeds, Department of Applied Mathematical Studies; 1997.

17. Brook BS, Pedley TJ: A model for time-dependent flow in (giraffe jugular) veins: uniform tube properties. I Biomech 2002, 35:95-107.

18. Anderson JD: Computational fluid dynamics : the basics with applications. New York, McGraw-Hill; 1995.

19. Milisic V, Quarteroni A: Analysis of lumped parameter models for blood flow simulations and their relation with ID models. Esaim-Mathematical Modelling and Numerical Analysis-Modelisation Mathematique Et Analyse Numerique 2004, 38:6I3-632.

20. Brook BS, Falle S, Pedley TJ: Numerical solutions for unsteady gravity-driven flows in collapsible tubes: evolution and rollwave instability of a steady state. Journal of Fluid Mechanics 1999 396:223-256.

21. Raju S, Green AB, Fredericks RK, Neglen PN, Hudson CA, Koenig K Tube collapse and valve closure in ambulatory venous pressure regulation: studies with a mechanical model. J Endovasc Surg 1998, 5:42-5I.

22. Neglen P, Raju S: Ambulatory venous pressure revisited. J Vasc Surg 2000, $31: 1206-1213$.

23. Willeput $R$, Rondeux $C$, De Troyer A: Breathing affects venous return from legs in humans. J Appl Physiol 1984, 57:971-976.

24. Abu-Yousef MM, Mufid M, Woods KT, Brown BP, Barloon T]: Normal lower limb venous Doppler flow phasicity: is it cardiac or respiratory? AJR Am J Roentgenol 1997, 169:1721-1725.

25. Fronek A, Criqui MH, Denenberg J, Langer RD: Common femoral vein dimensions and hemodynamics including Valsalva response as a function of sex, age, and ethnicity in a population study. J Vasc Surg 200I, 33:1050-1 056.

26. Dai G, Gertler JP, Kamm RD: The effects of external compression on venous blood flow and tissue deformation in the lower leg. J Biomech Eng 1999, I 2 I:557-564.
27. Buxton GA, Clarke N: Computational Phlebology: The Simulation of a Vein Valve. Journal of Biological Physics 2006, 32:507-52I.

28. Roark RJ, Young WC: Formulas for stress and strain. London, McGraw-Hill; 200I.

29. Berti G, Lonsdale G, Schmidt JG, Benker S, Hose DR, Fenner JW, Jones DM, Middleton SE, Wollny G: Grid simulation services for the medical community. International Journal of Computational Methods 2005

30. Fullana JM, Cros F, Flaud P, Zaleski S: Filling a collapsible tube. Journal of Fluid Mechanics 2003, 494:285-296.

31. Potter K: The effect of gravity on valve closure and vein collapse in the deep veins of the leg. Pressure and flow analysis. The University of Sheffield, Department of Medical Physics and Clinical Engineering; 2006.

32. Burnett J: The effect of gravity on valve closure and vein collapse in the deep veins of the leg. Image analysis. The University of Sheffield, Department of Medical Physics and Clinical Engineering; 2006.
Publish with Biomed Central and every scientist can read your work free of charge

"BioMed Central will be the most significant development for disseminating the results of biomedical research in our lifetime. "

Sir Paul Nurse, Cancer Research UK

Your research papers will be:

- available free of charge to the entire biomedical community

- peer reviewed and published immediately upon acceptance

- cited in PubMed and archived on PubMed Central

- yours - you keep the copyright 